\title{
Targeting the Tumor Stroma for Oral Cancer Therapy
}

ISSN: 2637-7764

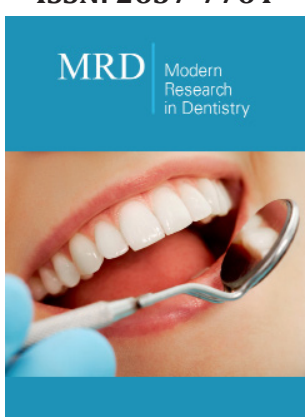

*Corresponding author: Takehito Ouchi, Department of Dentistry and Oral Surgery, Keio University School of Medicine, Shinjukuku, Tokyo, 160-8582, Japan

Submission: 監July 18, 2019

Published: 㭗July 30, 2019

Volume 4 - Issue 3

How to cite this article: Takehito $0^{*}$, Satoru M, Seiji A . Targeting the Tumor Stroma for Oral Cancer Therapy Mod Res Dent. 4(3). MRD.000586.2019. DOI: 10.31031/MRD.2019.04.000586

Copyright@ Takehito Ouchi, This article is distributed under the terms of the Creative Commons Attribution 4.0 International License, which permits unrestricted use and redistribution provided that the original author and source are credited.

\section{Takehito Ouchi ${ }^{1 *}$, Satoru Morikawa ${ }^{1}$ and Seiji Asoda ${ }^{1}$}

${ }^{1}$ Department of Dentistry and Oral Surgery, Keio University School of Medicine, Tokyo, Japan

\section{Opinion}

Histopathological diagnosis is commonly used to identify oral cancer. However, morphological diagnosis alone cannot clarify the biological malignancy and prognosis of this disease. Findings from cancer stem cell research and cancer genetic analysis help guide the design of cancer studies. In addition, the environmental niche, which includes cancer stem cells (CSCs) and/or cancer associated fibroblasts (CAFs), influences the behavior of the cancer cells and therefore the prognosis $[1,2]$. We utilize oral cancer samples to evaluate specific mesenchymal cell surface markers and to clarify cell properties and functions that may not be predicted by the pathological diagnosis [3]. Most cancers of the oral cavity are squamous cell carcinomas, with various degrees of malignancy. Spindle cell carcinoma (SpCC) is composed of a biphasic mixture of epithelial and mesenchymal cells [4]. SpCC is a subtype of poorly differentiated oral squamous cell carcinoma (OSCC) diagnosed by immunostaining of pathological specimens [5]. This traditional analysis can lead to diagnostic difficulties due to the range of histological patterns seen in SpCC. As it is a rarely diagnosed tumor, the characteristics of SpCC remain unclear [6]. Therefore, it is difficult for basic researchers and clinicians to determine the appropriate diagnosis and treatments. It is critical to understand the pathological and genetic characteristics of SpCC, including the gene expression patterns in the tumor microenvironment, to increase our understanding of this rare cancer.

Current oral cancer treatments are organ specific and mainly use reagents that target squamous cell carcinoma in the head and neck regions. For example, the EGFR-targeting reagent cetuximab is well known for its application in malignant disease of the head and neck. However, prior research demonstrated that cetuximab is not effective in cases of recurrent or advanced SpCC [6]. This underscores the necessity of identifying other cell components that can be targeted. Our group demonstrated that a cetuximab-resistant recurrent SpCC sample expressed the mesenchymal stem cells (MSCs) markers platelet-derived growth factor receptor $\alpha(P D G F R \alpha)$ and Nestin. Prospective cell analysis by using a flow cytometer revealed that recurrent SpCC cells expressed the CSC marker CD44v, which was identified in both EpCAM positive epithelial cells and PDGFR $\alpha$ positive mesenchymal cells. These results indicate that CSCs survived in both parenchymal and mesenchymal tissues. Cultured cells expressed legacy MSC markers such as CD73, CD90, and CD105, and they showed features such as colony forming, migration, and differentiation abilities. Our group demonstrated the utility of imatinib, which is known to inhibit the protein tyrosine kinase activity of BcrAbl and PDGFR $\alpha$, in targeting PDGFR $\alpha$-expressing stromal cells. Imatinib had a more potent inhibitory effect on the cultured cells and was more effective in inducing cell death relative to the cetuximab-treated group.

\section{Perspectives}

In our previous study, we evaluated the characteristics of cancer cells obtained from tissues in addition to conventional pathological diagnosis. By using a flow cytometer, it was possible to analyze the expression of markers several hours later. This strategy enables early prediction of the possible effects of chemotherapy and is expected to identify new markers and help develop drugs aimed at specific molecular targets. Analysis of the proliferation and migration abilities of the cancer cells, as well as drug response tests, can be used to further determine specific patient characteristics. This information, in combination with pathological analysis, helps inform diagnostic and treatment decisions and makes it possible to further 
elucidate the biological malignancy and prognosis of cancers as compared with pathological diagnosis alone. Our approach can contribute to the development of disease-specific individualized treatments that are essential for rare cancers with histologic types.

\section{Acknowledgment}

This work was supported by the Japan Society for the Promotion of Science KAKENHI, grant numbers 18K17034 (TO), 19K10172 (SM), and 19K10344 (SA). The authors declare no other potential conflicts of interest with respect to the authorship and/or publication of this article.

\section{References}

1. Plaks V, Kong N, Werb Z (2015) The cancer stem cell niche: how essential is the niche in regulating stemness of tumor cells? Cell Stem Cell 16(3): 225-238.
2. Melzer C, von der Ohe J, Lehnert H, Ungefroren H, Hass R (2017) Cancer stem cell niche models and contribution by mesenchymal stroma/stem cells. Mol Cancer 16(1): 28.

3. Ouchi T, Morikawa S, Shibata S, Takahashi M, Yoshikawa M, et al. (2018) Recurrent spindle cell carcinoma shows features of mesenchymal stem cells. J Dent Res 97(7): 779-786.

4. Gupta R, Singh S, Hedau S, Nigam S, Das BC, et al. (2007) Spindle cell carcinoma of head and neck: an immunohistochemical and molecular approach to its pathogenesis. J Clin Pathol 60(5): 472-475.

5. Leventon GS, Evans HL (1981) Sarcomatoid squamous cell carcinoma of the mucous membranes of the head and neck: a clinicopathologic study of 20 cases. Cancer 48(4): 994-1003.

6. Watson RF, Chernock RD, Zhang KH, Michel LS, Adkins DR, et al. (2015) Epidermal growth factor receptor expression in spindle cell carcinomas of the head and neck. Head Neck Pathol 9(3): 360-368. 